Meta

Journal des tradlucteurs

Translators' Journal

\title{
Étude de l'emprunt boxer dans le domaine vestimentaire
}

\section{Geneviève Mareschal}

Volume 32, numéro 4, décembre 1987

URI : https://id.erudit.org/iderudit/003572ar

DOI : https://doi.org/10.7202/003572ar

Aller au sommaire du numéro

Éditeur(s)

Les Presses de l'Université de Montréal

ISSN

0026-0452 (imprimé)

1492-1421 (numérique)

Découvrir la revue

Citer cet article

Mareschal, G. (1987). Étude de l'emprunt boxer dans le domaine vestimentaire. Meta, 32(4), 438-442. https://doi.org/10.7202/003572ar d'utilisation que vous pouvez consulter en ligne.

https://apropos.erudit.org/fr/usagers/politique-dutilisation/ 


\section{ÉTUDES TERMINOLOGIQUES ET LINGUISTIQUES}

\section{ÉTUDE DE L'EMPRUNT BOXER DANS LE DOMAINE VESTIMENTAIRE}

Tributaire d'une mode vestimentaire, l'emprunt à l'anglais boxer a fait son entrée dans la langue française au début des années 70 . Bien qu'il ne figure dans aucun dictionnaire français de la langue courante, ni même de la langue contemporaine, boxer s'est rapidement forgé une place dans le lexique vestimentaire français. Son emploi dans les publications francophones', d'une part, et l'extension sémantique acquise par ce terme au sein de la langue d'accueil, d'autre part, témoignent en ce sens.

Or, il est assez curieux de constater que boxer n'existe pas en anglais en tant qu'unité lexicale autonome dans le domaine vestimentaire. Renvoyant étymologiquement au sport de la boxe et à celui qui le pratique, il est généralement associé à un autre terme vestimentaire et sert à en préciser le modèle ou le style. C'est ainsi qu'on trouve en anglais des syntagmes tels que boxer trunks, boxer waist, où boxer détermine le nom qu'il accompagne, et, quelle que soit l'unité syntagmatique ainsi formée, le rôle sémantique joué par ce déterminant est d'apparenter l'article vestimentaire qu'il détermine au style de la culotte portée par les boxeurs, c'est-à-dire ample et à taille élastique froncée. Le rôle de déterminant que boxer est souvent appelé à jouer explique probablement la non-consignation comme entrées lexicographiques distinctes de la plupart des syntagmes qu'il sert à former. Seul boxer shorts, qui désigne un sous-vêtement masculin et est parfois abrégé en boxers dans la langue familière, a atteint un degré suffisant de lexicalisation pour avoir droit à une entrée autonome au dictionnaire anglais.

Le passage de l'emprunt boxer au français a non seulement donné lieu à des changements sémantiques et morphologiques, mais il s'est aussi opéré de façon inégale au sein de la francophonie. Le Québec et l'Europe francophone n'ont pas accueilli et intégré l'emprunt de la même manière, et cette différence s'affirme à la fois sur le plan sémantique et sur le plan morphologique. Alors que le français québécois est resté conforme à l'anglais au niveau du signifié, le français européen, lui, l'est plutôt resté au niveau du signifiant.

Le tableau ci-dessous illustre cette intégration et permet d'analyser et de comparer l'évolution sémantique, morphologique et géographique de boxer en français.

Définition : Caleçon court et ample, plissé à la taille par une ceinture élastique (son style s'apparente à celui de la culotte de boxeur) (Dupré 1980).

Anglais : boxer shorts, parfois abrégé en boxers

Français (Québec) : caleçon boxeur (ex. 1)

Français (Europe) : (correspond plus ou moins au caleçon court)

Définition : Style ou coupe vestimentaire rappelant ceux de la culotte de boxeur, c'est-àdire ample et froncée à la taille par une ceinture élastique.

Anglais : boxer + déterminé. Ex. : boxer trunks, boxer waist

Français (Québec) : déterminé + boxer. Ex. : short boxeur (Dupré 1980), ceinture boxeur (Dupré 1980).

Français (Europe) : déterminé + boxer. Ex. : ceinture boxer (ex. 2), pantalon boxer (ex. 3). 
Définition : Sous-vêtement couvrant le bas du corps, pourvu de courtes jambes moulantes cachant le haut des cuisses.

Anglais : (modèle qui ne semble pas répandu)

Français (Québec) : (modèle qui ne semble pas répandu)

Français (Europe) : boxer (ex. 4, 5), culotte(-)boxer (ex. 6), slip(-)boxer (ex. 7)

Définition : Culotte de bain pourvue de courtes jambes moulantes couvrant le haut des cuisses.

Anglais : (contour-leg swimsuit) ou (square-leg swimsuit)

Français (Québec) : (pour les femmes : culottes à jambes droites); (pour les hommes : caleçon de bain, par opposition à slip de bain)

Français (Europe) : boxer (ex. 8), boxer(-)short (ex. 9, 10), culotte(-)boxer (ex. 11), slip(-)boxer (ex. 12)

Les exemples numérotés entre parenthèses renvoient à l'annexe.

Contrairement à l'anglais où boxer n'a qu'un sens ${ }^{2}$ dans le lexique vestimentaire, le français lui en reconnaît deux : 1) caleçon court et ample, plissé à la taille par une ceinture élastique, dont le style rappelle la culotte portée par les boxeurs (déf. 1 et 2$)^{3}$ et 2) article vestimentaire couvrant le bas du corps, pourvu de courtes jambes moulantes cachant le haut des cuisses (déf. 3 et 4$)^{3}$. De ces deux sens, le premier est emprunté à l'anglais et le second est propre au français. Boxer a par conséquent acquis dans la langue d'accueil un nouveau sens, qui n'est pas attesté dans la langue d'origine. Il y a eu extension sémantique en français et boxer s'y est vu attribuer, en plus de l'évocation de la culotte de boxeur et de sa coupe, empruntée à l'anglais, la désignation d'un article vestimentaire d'une autre coupe et d'un autre style, toujours destiné cependant à couvrir le bas du corps. Les définitions 3 et 4 décrivent ce nouvel emploi et ne diffèrent entre elles que par le rôle joué par le vêtement en question, l'un se situant au niveau sousvestimentaire (déf. 3 ) et l'autre, au niveau vestimentaire (déf. 4).

Sur le plan morphologique, trois formes ont cours en français : boxer, boxeur et boxer(-)short. Dans le cas de boxer, il y a en fait lieu de distinguer deux formes : l'une en tant qu'unité lexicale autonome, l'autre en tant qu'élément de composition. C'est d'ailleurs en tant qu'élément de composition que boxer offre en français le rapport le plus étroit avec la langue dont il est issu sur le plan morphosyntaxique et, dans le cas de la définition 2, sur le plan sémantique. En effet, en anglais comme en français, boxer sert de cléterminant à un article vestimentaire ou à une partie d'article vestimentaire dont il vient préciser le style, en l'apparentant entre autres à la culotte de boxeur, et se combine avec ce déterminé pour former une unité syntagmatique. Comme en anglais, il ne jouit pas d'une existence autonome et il n'acquiert sa pleine valeur lexicale qu'en composition.

Élément de composition au départ, la forme boxer a toutefois acquis en français une autonomie de fonctionnement et un sens propre. Boxer est devenu une nouvelle unité lexicale française (déf. 3 et 4), avec toutes les prérogatives que cela comporte, dont celle d'entrer en composition avec d'autres unités lexicales et de former avec elles de nouvelles unités syntagmatiques. La culotte(-)boxer et le slip(-)boxer des définitions 3 et 4 en sont une illustration, boxer renvoyant dans ces composés au nouveau sens acquis en français. Il est toutefois intéressant de noter que, dans ces syntagmes, boxer a gardé le rôle de déterminant qu'il avait en tant qu'élément de composition dans la définition 2, et non de déterminé, comme on aurait pu s'y attendre étant donné l'existence autonome 
nouvellement acquise par cet emprunt. On aurait tout aussi logiquement pu avoir boxer-slip et boxer-culotte, avec slip et culotte comme déterminants et boxer comme déterminé, que le contraire. Il reste que le trait sémique principal de la nouvelle unité lexicale est essentiellement celui d'une forme, d'un modèle et qu'à ce titre, elle se voit presque automatiquement confinée au rôle de déterminant en composition.

La forme boxeur résulte d'une francisation de l'anglais boxer par adaptation morphologique du suffixe -er en -eur. La similitude phonique et sémantique des deux suffixes d'agent favorise cette substitution. En outre, le mot " boxeur " existe depuis longtemps en français pour désigner celui qui pratique le sport de la boxe, et a certainement concouru à l'adoption de boxeur dans le lexique vestimentaire. On peut même se demander si, plutôt qu'à un processus de francisation, le français n'a pas eu recours à un processus de traduction en retenant boxeur comme équivalent de boxer dans le domaine qui nous occupe. Cette hypothèse a d'autant plus de poids que l'emploi de boxeur se limite géographiquement au Québec où la pratique du calque ${ }^{4}$ est beaucoup plus répandue qu'ailleurs dans la francophonie quand il s'agit d'emprunter à l'anglais's. De plus, dans les exemples que nous avons relevés, la forme boxeur n'est pas employée de façon autonome, mais en composition. Or, l'unité syntagmatique constitue le lieu privilégié du calque. Il nous apparaît donc entièrement défendable de considérer caleçon boxeur et ceinture boxeur comme des calques de boxeur shorts et boxer waist et de voir dans l'adoption de la forme boxeur un réflexe de traduction plutôt que de francisation, encore que l'on aboutisse au même résultat.

La forme boxer(-)short, morphologiquement très proche de l'anglais boxer shorts, a subi au cours de son passage de l'anglais au français une adaptation morphogrammaticale destinée à la rendre plus conforme au système du français. En effet, short est, en français, un nom singulier et ne comporte pas de $-s$ final, contrairement à l'anglais qui utilise toujours la forme du pluriel pour désigner un vêtement avec des jambes (par ex. trousers, briefs, dungarees). Par analogie avec le français, il y a eu suppression du $-s$ final de l'anglais, la forme originelle ayant été ressentie en français comme un véritable pluriel de pluralité. Par ailleurs, bien que l'usage soit hésitant; l'addition d'un trait d'union entre boxer et short a pour effet de mieux figer le syntagme et de lui conférer en français le même degré de cohésion et de lexicalisation que celui perçu en anglais. Sur le plan sémantique toutefois, boxer(-)short n'est aucunement l'équivalent de boxer shorts puisqu'il ne renvoie qu'au sens acquis directement en français par boxer (déf. 3 et 4). L'emprunt en l'occurrence se situe uniquement au niveau du signifiant, d'ailleurs adapté au système morphologique du français, et en aucune façon au niveau du signifié.

Sur le plan de la distribution géographique, deux éléments dominants ressortent du tableau.

1. Le français européen, conformément au préjugé favorable qu'il entretient à l'endroit de l'anglicisme, n'hésite pas à emprunter boxer et ses composés, mais cette adoption se limite presque exclusivement au signifiant, puisque dans deux cas sur trois (déf. 3 et 4), le signifié auquel renvoie boxer en français est inconnu de l'anglais.

2. Le français québécois, plus rebelle à l'emprunt et principalement à l'emprunt de signifiant, rejette boxer en tant que signifiant et lui substitue une forme plus française boxeur, mais conserve intact le signifié anglais qu'il recouvre et n'adopte pas le nouveau sens acquis par boxer en français.

Il convient également de noter que l'aire sémantique de boxer et de ses composés et surtout de boxer-short en français européen s'est étendue au sexe féminin, alors qu'en anglais et en français québécois, cette unité lexicale s'applique principalement au sexe masculin et, dans le cas de boxer shorts et de caleçon boxeur, exclusivement à celui-ci. 
Pour terminer, il serait peut-être intéressant d'imaginer comment cet emprunt pourrait véritablement s'intégrer au lexique vestimentaire français si l'usage venait à lui confirmer son droit à l'existence. Il serait par exemple possible de réserver la forme française existante boxeur à la désignation du sens couvert par les définitions 1 et 2 , forme qui figurerait uniquement en tant que déterminant syntagmatique dans des composés lexicaux sans joncteur (ex. : short boxeur) et qui aurait l'avantage de rester proche étymologiquement et sémantiquement du terme anglais, et de réserver le signifiant anglais boxer à l'expression du sens couvert par les définitions 3 et 4 . On créerait ainsi une nouvelle unité lexicale en français qui, bien que d'origine morphologique anglais (on pourrait d'ailleurs sans aucun mal adopter une prononciation française [boks r] sur le modèle de imper, reporter, etc.), aurait un sens exclusivement français.

GENEVIÈVE MARESCHAL Université d'Ottawa, Ottawa, Canada

Notes

1. Une liste d'exemples, relevés dans quatre aires de la francophonie, est donnée en annexe.

2. Nous utilisons " sens " comme synonyme d" acception", sans aucune référence à une théorie linguistique particulière.

3. Nous renvoyons aux définitions du tableau ci-dessus.

4. Par "calque", nous entendons le transfert, sous une forme traduite, d'une unité lexicale et de ses constituants d'une langue dans une autre.

5. Il suffit de comparer un dictionnaire d'anglicismes québécois et d'anglicismes européens pour le constater.

\section{Annexe}

Les exemples ci-dessous ont été relevés dans des catalogues de vente par correspondance entre 1976 et 1984 .

\section{Définition 1}

1. Caleçon boxeur Perma-Prest en broadcloth de polyester et coton à laver, sécher machine, sans repassage. Braguette, taille élastique. (Québec)

\section{Définition 2}

2. Le caleçon tissu quadrillé, $65 \%$ polyester, $35 \%$ coton. Ceinture boxer fermée par deux boutons. Lavage à $40^{\circ}$. (Belgique)

3. Pyjama judo $65 \%$ polyester et $35 \%$ coton. Bordure unie à l'encolure en $\mathrm{V}$, au boutonnage, au bord des poches plaquées et au bas des manches. Pantalon boxer uni, avec braguette fermée par un bouton. (France)

\section{Définition 3}

4. Boxer en stretch $80 \%$ coton, $20 \%$ polyamide Helanca. Élastique à la ceinture et aux jambes. (France)

5. Soutien-gorge Fr. 19,90

Gaine-culotte Fr. 39,50

Gaine Fr. 29,50

Slip Fr. 29,50

Boxer Fr. 24,50

(Suisse)

6. Culotte boxer assortie aux 2 soutiens-gorge. $83 \%$ polyamide, $17 \%$ élasthane. Picot élastique à la taille, agrémenté d'une fine dentelle. Fond doublé coton. (France)

7. Slip-boxer Sarlana idéal à porter sous un pantalon car il galbe et affine sans démarcations disgracieuses. Jersey renforcé Elasthan. (Suisse) 


\section{Définition 4}

8. Maillot 2 pièces. Le soutien-gorge : coques incorporées doublées, forme brassière à nouer au cou. Le boxer : élastique à la taille et aux jambes. (France)

9. De 2 à 16 ans, le maillot que l'on verra sur toutes les plages s'appelle "boxer-short ". Plus grand qu'un slip, il permet de bronzer ses jambes sans se montrer indiscret au moindre mouvement. (France)

10. Le boxer short. En $62 \%$ acrylique, $38 \%$ polyamide. Large élastique sous tunnel à la taille. Poche gousset. Slip intérieur en $100 \%$ polyamide. (Belgique)

11. Maille Dropnyl Nylfrance Hélanca $100 \%$ polyamide. Culotte boxer marine rattachée par une pointe au soutien-gorge imprimé à coques amovibles. (France)

12. Un soutien-gorge bandeau maintenu par une bretelle nouée autour du cou. Petit slip boxer. Bilytis, $990 \mathrm{~F}$ env. (Belgique)

\section{BIBLIOGRAPHIE}

Catalogues de vente par correspondance :

Jelmolispour la Suisse

La Redoute pour la France

Trois-Suisses pour la Belgique"

Sears pour le Québec

DUPRÉ, Céline (1980) : Vocabulaire de l'habillement, Québec, Éditeur officiel, 195 p. 